\title{
Zeta-regularization of arithmetic sequences
}

\author{
Jean-Paul Allouche ${ }^{1, *}$ \\ ${ }^{1}$ CNRS, IMJ-PRG, Sorbonne Université \\ 4 Place Jussieu \\ F-75252 Paris Cedex 05 France
}

\begin{abstract}
Is it possible to give a reasonable value to the infinite product $1 \times 2 \times 3 \times \cdots \times n \times \cdots$ ? In other words, can we define some sort of convergence of the finite product $1 \times 2 \times 3 \times \cdots \times n$ when $n$ goes to infinity? One way is to relate this product to the Riemann zeta function and to its analytic continuation. This approach leads to: $1 \times 2 \times 3 \times \cdots \times n \times \cdots=\sqrt{2 \pi}$. More generally the "zeta-regularization" of an infinite product consists of introducing a related Dirichlet series and its analytic continuation at 0 (if it exists). We will survey some properties of this generalized product and allude to applications. Then we will give two families of possibly new examples: one unifies and generalizes known results for the zeta-regularization of the products of Fibonacci, balanced and Lucas-balanced numbers; the other studies the zeta-regularized products of values of classical arithmetic functions. Finally we ask for a possible zeta-regularity notion of complexity.
\end{abstract}

\section{Introduction}

Mathematicians and physicists like to bypass the "rules" that objects in their fields have to satisfy: they often want to explore whether there is something interesting beyond these rules. For example, they "invented" negative numbers to give a meaning to $3-5$. They legitimated expressions like $2 / 3, \sqrt{2}$, or $\sqrt{-1}$. They even introduced expressions like $\sum_{n \geq 1} 1 / n^{2}$. Another example is the theory of distributions: does there exist a function which is zero everywhere except at 0 where its value is $\infty$, and whose integral on the real line is equal to 1 ? Sure! it evens has a name (the Dirac function of course). In the same spirit, how is it possible to give a reasonable meaning to non-convergents infinite products, e.g., $1 \times 2 \times 3 \times \cdots \times n \times \cdots$ ? This is a typical example of a product for which there exists a socalled "zeta-regularization", which assigns the value $\sqrt{2 \pi}$ to this product.

In the sequel, we will first recall a "natural" definition of zeta-regularizations of infinite products, and give some properties and "classical" examples. For this part the reader is referred to the references given at the end, and to the references given in [1]. Then we will give two families of examples, one providing a unified treatment of Fibonacci-like sequences; the other showing zetaregularized products that we did not find in the literature, where arithmetic functions like $d(n)$, the number of divisors of the integer $n$, enter the picture. Finally we will mention our (still unsuccessful) attempts to concoct a complexity measure from the zeta-regularized product of sequences of integers.

*e-mail: jean-paul.allouche@imj-prg.fr
It is worth noting that zeta-regularization of infinite products is used in physics, for example to define the determinant of certain infinite-dimensional operators. We cite only one reference, namely a 1977 paper by Hawking [10], which, in particular, gives a way of calculating the Casimir effect between two parallel reflecting planes.

\section{Definition and first properties}

\subsection{The regularized product}

Suppose that $\Lambda=\left(\lambda_{i}\right)_{1 \leq i \leq n}$ is a finite sequence of positive real numbers. Define

$$
\zeta_{\Lambda}(s)=\sum_{i=1}^{n} \frac{1}{\left(\lambda_{i}\right)^{s}} .
$$

We clearly have

$$
\zeta_{\Lambda}^{\prime}(0)=-\sum_{i=1}^{n} \log \lambda_{i} \text { i.e., } \log \prod_{i=1}^{n} \lambda_{i}=-\zeta_{\Lambda}^{\prime}(0)
$$

which can be written

$$
\prod_{i=1}^{n} \lambda_{i}=e^{-\zeta_{\Lambda}^{\prime}(0)}
$$

Now suppose that $\Lambda=\left(\lambda_{i}\right)_{i \geq 1}$ is an infinite non-decreasing sequence of positive real numbers. Let $\zeta_{\Lambda}(s)$ the associated Dirichlet series, i.e.,

$$
\zeta_{\Lambda}(s)=\sum_{i \geq 1} \frac{1}{\left(\lambda_{i}\right)^{s}} .
$$

By analogy with what precedes, we give the following definitions (see, [6] or [25, p. 96-97]): 
Definition 1 Suppose that $\zeta_{\Lambda}(s)$ converges for $\mathfrak{R}$ s large enough. If $\zeta_{\Lambda}(s)$ has a meromorphic continuation to a region containing 0 with at most simple poles, and has no pole at $s=0$, then the zeta-regularized product of the $\lambda_{i}$ 's, denoted $\prod_{i \geq 1} \lambda_{i}$, is defined by

$$
\prod_{i \geq 1} \lambda_{i}:=e^{-\zeta_{\Lambda}^{\prime}(0)}
$$

This definition clearly coincides with the usual product if the set $\Lambda$ is finite.

A more general definition is given, e.g., in $[12,14]$ :

Definition 2 Suppose that $\zeta_{\Lambda}(s)$ converges for $\mathfrak{R}$ s large enough. If $\zeta_{\Lambda}(s)$ has a meromorphic continuation to a region containing 0 and a pole at 0 , then the zeta-regularized product of the $\lambda_{i}$ 's (still denoted $\prod_{i \geq 1} \lambda_{i}$ ), is defined by

$$
\prod_{i \geq 1} \lambda_{i}:=e^{-\operatorname{Re} s \frac{\zeta \Lambda(s)}{s^{2}}} .
$$

Of course both definitions coincide if $\zeta_{\Lambda}(s)$ satisfies the hypotheses in the first definition.

Remark 3 Several symbols occur in the literature for zeta-regularized products and/or their generalizations $\left(\Pi_{R} \Pi_{z} \hat{\prod} \sqcap \boxminus \Pi\right)$, see $[11,14,17$, 22]. Here we keep the same notation (the symbol $\square$ ) for all variations on the zeta-regularized product. To type these symbols in $\mathrm{LT}_{\mathrm{E}} \mathrm{X}$, see the very useful link: https://tex.stackexchange.com/questions/ 50669/regularized-product-symbol.

The following properties of $\square$ are easy to prove.

\section{Proposition 4}

(i) If $\Lambda=\left\{\lambda_{1}, \lambda_{2}, \cdots, \lambda_{k}, \lambda_{k+1} \ldots\right\}$ with positive $\lambda_{i}$ 's, then $\prod_{i=1}^{\infty} \lambda_{i}=\prod_{j=1}^{k} \lambda_{j} \prod_{i=k+1}^{\infty} \lambda_{i}$.

(ii) If If $A \cup B$ is a partition of the positive integers, then $\prod_{i=1}^{\infty} \lambda_{i}=\prod_{i \in A} \lambda_{i} \prod_{i \in B} \lambda_{i}$.

(iii) If $d$ is a positive integer, then $\square \lambda_{i}^{d}=\left(\coprod \lambda_{i}\right)^{d}$.

(iv) For $a>0, \prod_{i=1}^{\infty}\left(a \lambda_{i}\right)=a^{\zeta_{\Lambda}(0)} \prod_{i=1}^{\infty} \lambda_{i}$.

Remark 5 In general $\square\left(\lambda_{i} \mu_{i}\right) \neq\left(\coprod \lambda_{i}\right)\left(\coprod \mu_{i}\right)$.

\section{Classical examples}

We give some classical examples.
* The Kurokawa-Wakayama formula [14] reads

$$
\begin{aligned}
& \prod_{n \geq 0}\left((n+x)^{m}-y^{m}\right)=\frac{(2 \pi)^{m / 2}}{\prod_{\xi^{m}=1} \Gamma(x-\xi y)} \text { for } m=1,2, \ldots \\
& \prod_{n \geq 0}\left((n+x)^{m}+y^{m}\right)=\frac{(2 \pi)^{m / 2}}{\prod_{\xi^{m}=1} \Gamma(x+\xi y)} \text { for } m=1,3, \ldots \\
& \prod_{n \geq 0}\left((n+x)^{m}+y^{m}\right)=\frac{(2 \pi)^{m / 2}}{\prod_{\xi^{m}=-1} \Gamma(x+\xi y)} \text { for } m=2,4, \ldots
\end{aligned}
$$

* The case $m=2$ of the last formula above is due to Lerch. It reads

$$
\prod_{n \geq 0}\left((n+x)^{2}+y^{2}\right)=\frac{2 \pi}{\Gamma(x+i y) \Gamma(x-i y)} .
$$

In particular, by taking $y=0$, then $y=0, x=1$,

$\prod_{n \geq 0}(n+x)=\frac{\sqrt{2 \pi}}{\Gamma(x)}$ and $\prod_{n \geq 1} n=\sqrt{2 \pi}$.

Thus

$\prod_{n \geq 1}(2 n)=\sqrt{\pi}$ and $\prod_{n \geq 1}(2 n+1)=\sqrt{2}$.

Other examples deduced from the Lerch formula or from the Kurokawa-Wakayama formula are:

$\prod_{n \geq 0}\left(n^{2}+1\right)=e^{\pi}-e^{-\pi}$
$\prod_{n \geq 0}\left(n^{2}-n+1\right)=e^{\pi \frac{\sqrt{3}}{2}}+e^{-\pi \frac{\sqrt{3}}{2}}$
$\prod_{n \geq 2}\left(n^{4}+1\right)=\cosh (\pi \sqrt{2})-\cos (\pi \sqrt{2})$
$\prod_{n \geq 2}\left(n^{4}-1\right)=\pi \sinh (\pi)$.

* Some basic "arithmetic" examples are:

$\prod_{n \geq 0} a^{n}=a^{-1 / 12}$ for $a>1$. (Compare with the famous "Ramanujan sum" $\sum_{n \geq 1} n=-1 / 12$, see, e.g., [27].)

$\prod_{n \in S} n=2 \pi$, where $S$ is the set of squarefree integers. (Note that we also have $\prod_{n \geq 1} n^{2}=2 \pi$.)

$\prod_{n \geq 1} n^{n}=e^{-\zeta^{\prime}(-1)}=A e^{-1 / 12}$, where $A=e^{-\zeta^{\prime}(-1)+1 / 12}$ is known as the Glaisher-Kinkelin constant.

\section{Remark 6}

* From, e.g., [4, p. 6] one has (compare with Remark 5):

$$
\frac{\prod_{n \geq 2}\left(n^{4}-1\right)}{\prod_{n \geq 2}\left(n^{4}+1\right)}=\prod_{n \geq 2} \frac{n^{4}-1}{n^{4}+1} .
$$

* To prove $\prod_{n \in s a f} n=2 \pi$, let $\mu$ be the Möbius function. From, e.g., [9], $Q(s):=\sum_{n \geq 1} \frac{|\mu(n)|}{n^{s}}=\frac{\zeta(s)}{\zeta(2 s)}$ for $\mathfrak{R} s>1$. Then $Q^{\prime}(0)=-\zeta^{\prime}(0) / \zeta(0)=-\frac{-\log (2 \pi) / 2}{-1 / 2}=-\log (2 \pi)$. 
* The example $\prod_{n \geq 1} n^{n}=e^{-\zeta^{\prime}(-1)}$ might need some explanation: it should be considered as the zetaregularized product of the sequence of integers

$$
\underbrace{1}_{1 \text { factor }} \underbrace{22}_{2 \text { factors }} \underbrace{333}_{3 \text { factors }} \cdots \underbrace{n n \ldots n}_{n \text { factors }} \cdots
$$

More generally, if $\left(a_{n}\right)$ is a sequence of integers, then $\prod_{n \geq 1} n^{a_{n}}$ should be considered as the zeta-regularized product of the following sequence of integers

$$
\Lambda=\underbrace{1 \ldots 1}_{a_{1} \text { factors }} \underbrace{2 \ldots 2}_{a_{2} \text { factors }} \underbrace{3 \ldots 3}_{a_{3} \text { factors }} \cdots \underbrace{n \ldots n}_{a_{n} \text { factors }} \cdots
$$

with associated Dirichlet series $\sum_{n \geq 1} \frac{a_{n}}{n^{s}}=\zeta_{\Lambda}(s)$.

\section{Fibonacci and Fibonacci-like numbers}

The zeta-regularized product of the Fibonacci numbers (i.e., the sequence A000045 $=0,1,1,2,3 \ldots$ in the OEIS [20]) and the zeta-regularized product of the balancing and Lucas-balancing numbers (i.e., the sequences A001109 and A001541 in [20]) are computed in [8, 13] and in [7]. Of course the proofs involve the zeta functions associated with these sequences of integers. We give here a unified proof based on "explicit" expressions for the meromorphic continuations of these sequences. Our approach is similar to the ones given, e.g., in $[19,23]$ or, in a different context, in [2] (also see, e.g., [1, 3, 26], and [5] which gives interesting historical remarks).

Theorem 7 Let $a$ and $b$ be two rational integers with $a^{2}-4 b>0$ such that its two (real) roots $\lambda$ and $\lambda^{\prime}$ satisfy $\lambda>1$ and $\left|\lambda^{\prime}\right|=|b| / \lambda<1$. Let $\left(X_{n}\right)_{n \geq 0}$ be a sequence of integers that satisfies the recurrence equation $X_{n+2}=a X_{n+1}-b X_{n}$, for all $n \geq 0$. Let $X_{n}=\alpha \lambda^{n}+\beta \lambda^{\prime n}$. We let $\gamma=-\beta / \alpha$ and we suppose that $\alpha>0$ and that $|\gamma b| / \lambda^{2}<1$. Define $\zeta_{X}(s)=\sum_{n \geq 1} 1 / X_{n}^{s}$. Then $\zeta_{X}$ has a meromorphic continuation given by

$$
\frac{1}{\alpha^{s}\left(\lambda^{s}-1\right)}+\frac{1}{\alpha^{s}} \sum_{k \geq 1}\left(\begin{array}{c}
s+k-1 \\
k
\end{array}\right) \frac{\gamma^{k} \beta^{k}}{\lambda^{2 k+s}-b^{k}} .
$$

Furthermore the coefficient of $s$ in the Laurent series expansion of this continuation in the neighborhood of $s=0$ is equal to

$$
\frac{\log \alpha}{2 \log \lambda}+\frac{1}{2} \log \alpha+\frac{1}{12} \log \lambda-\log \prod_{n \geq 1}\left(1-\gamma\left(b \lambda^{-2}\right)^{n}\right) .
$$

Proof. We have $X_{n}=\alpha \lambda^{n}+\beta \lambda^{\prime n}=\alpha \lambda^{n}\left(1-\gamma\left(b \lambda^{-2}\right)^{n}\right)$, hence

$$
\begin{aligned}
\zeta_{X}(s) & =\sum_{n \geq 1} \frac{1}{X_{n}^{s}}=\frac{1}{\alpha^{s}} \sum_{n \geq 1} \frac{1}{\lambda^{n s}}\left(1-\gamma\left(b \lambda^{-2}\right)^{n}\right)^{-s} \\
& =\frac{1}{\alpha^{s}} \sum_{n \geq 1} \frac{1}{\lambda^{n s}} \sum_{k \geq 0}\left(\begin{array}{c}
s+k-1 \\
k
\end{array}\right) \gamma^{k}\left(b \lambda^{-2}\right)^{n k} \\
& =\frac{1}{\alpha^{s}} \sum_{n \geq 1} \frac{1}{\lambda^{n s}}+\frac{1}{\alpha^{s}} \sum_{n \geq 1} \frac{1}{\lambda^{n s}} \sum_{k \geq 1}\left(\begin{array}{c}
s+k-1 \\
k
\end{array}\right) \gamma^{k}\left(b \lambda^{-2}\right)^{n k} \\
& =\frac{1}{\alpha^{s}\left(\lambda^{s}-1\right)}+\frac{1}{\alpha^{s}} \sum_{n \geq 1} \frac{1}{\lambda^{n s}} \sum_{k \geq 1}\left(\begin{array}{c}
s+k-1 \\
k
\end{array}\right) \gamma^{k}\left(b \lambda^{-2}\right)^{n k} \\
& =\frac{1}{\alpha^{s}\left(\lambda^{s}-1\right)}+H(s) .
\end{aligned}
$$

The function $H$ is holomorphic, so that we have the meromoprhic continuation of $\zeta_{X}$, with poles located at $s$ such that $\lambda^{s}=1$. Furthermore $H(0)=0$ (since $\left(\begin{array}{c}s+k-1 \\ k\end{array}\right)=$ $s(s+1) \ldots(s+k-1) / k !)$. So that

$$
\begin{aligned}
H^{\prime}(0) & =\lim _{s \rightarrow 0} \frac{H(s)}{s}=\sum_{n \geq 1} \sum_{k \geq 1} \frac{1}{k} \gamma^{k}\left(b \lambda^{-2}\right)^{n k} \\
& =-\log \prod_{n \geq 1}\left(1-\gamma\left(b \lambda^{-2}\right)^{n}\right) .
\end{aligned}
$$

On the other hand, a quick computation shows that the coefficient of $s$ in the Laurent series expansion of $\frac{1}{\alpha^{s}\left(\lambda^{s}-1\right)}$ is

$$
\frac{\log \alpha}{2 \log \lambda}+\frac{1}{2} \log \alpha+\frac{1}{12} \log \lambda
$$

Corollary 8 With the notation of Theorem 7 we have

$$
\prod_{n \geq 1} X_{n}=\alpha^{-1 / 2} \lambda^{-1 / 12} e^{-\log ^{2} \alpha / 2 \log \lambda} \prod_{n \geq 1}\left(1-\gamma\left(b \lambda^{-2}\right)^{n}\right) .
$$

In particular we have:

* (see [13]) Let $F_{n}$ be the Fibonacci numbers, defined by $F_{0}=0, F_{1}=1$, and for all $n \geq 0, F_{n+2}=F_{n+1}+F_{n}$. Let $\varphi=\frac{1+\sqrt{5}}{2}$. Then

$$
\prod_{n \geq 1} F_{n}=\left(\frac{5^{1 / 4} e^{-\left(\log ^{2} 5\right) /(8 \log \varphi)}}{\varphi^{1 / 12}}\right) \prod_{n \geq 1}\left(1-\left(-\varphi^{2}\right)^{-n}\right) .
$$

* (see [7]) Let $B_{n}$ and $C_{n}$ be the balancing and Lucasbalancing numbers, defined by $B_{0}=0, B_{1}=1$, and for all $n \geq 0, B_{n+2}=6 B_{n+1}-B_{n}$, respectively $C_{0}=0, C_{1}=3$ and for all $n \geq 0, C_{n+2}=6 C_{n+1}-C_{n}$. Let $\mu=3+\sqrt{8}$. Then

$$
\prod_{n \geq 1} B_{n}=\left(\frac{2^{5 / 4} e^{-\left(\log ^{2} 32\right) /(8 \log \mu)}}{\mu^{1 / 12}}\right) \prod_{n \geq 1}\left(1-\mu^{-2 n}\right)
$$

and

$$
\prod_{n \geq 1} C_{n}=\left(\frac{\sqrt{2} e^{-\left(\log ^{2} 2\right) /(2 \log \mu)}}{\mu^{1 / 12}}\right) \prod_{n \geq 1}\left(1+\mu^{-2 n}\right) .
$$

Remark 9 Note that there is a misprint in [13]: $(-\varphi)^{-2 n}$ should be replaced with $\left(-\varphi^{2}\right)^{-n}$.

\section{Some more arithmetic examples}

The first result of this section addresses the product of all primes. The definitions above for zeta-regularized products cannot be used: the Dirichlet series $\sum_{p}$ prime $1 / p^{s}$ cannot be extended meromorphically to a neighborhood of 0 since the imaginary axis is its natural boundary [16]. But, extending once more the zeta-regularization of a product, the notion of "super-regularization" was defined in [18] (also see [24]) yielding:

$$
\prod_{p \text { prime }} p=4 \pi^{2}
$$


A second arithmetic example is the product of all odious numbers. Recall that an integer is said to be odious if its binary expansion contains an odd number of 1's and evil otherwise. One has [1]:

Let $\Lambda=1247811131416 \ldots$ be the sequence of odious numbers (sequence A000069 in the OEIS [20]). Then

$$
\prod_{n \in \Lambda} n=\pi^{1 / 4} \sqrt{2 \varphi e^{-\gamma}}
$$

where $\gamma$ and $\varphi$ are respectively the Euler-Mascheroni and the Flajolet-Martin constants.

The following examples might not be in the literature. They involve arithmetic functions (as was previously the case with the Dirichlet series $\sum \frac{|\mu(n)|}{n^{5}}$; see both parts of Remark 6 above) and largely use the catalog of Dirichlet series [9] and the references therein, from which it is easy to compute their derivatives at 0 . We give only a few examples; several other examples can be deduced from [9] and its references.

Theorem 10 Let $d(n)$ be the number of divisors of the integer $n, \sigma(n)$ be the sum of the divisors of the integer $n$, and $\varphi(n)$ be the Euler totient function. Let $\theta(n)$ be the number of ordered paris $a, b$ of positive integers such that $n=a b$ and $\operatorname{gcd}(a, b)=1$ (hence $\theta(n)=2^{\omega(n)}$ where $\omega(n)$ is the number of distinct prime factors of $n$ ). Let $\zeta$ be the Riemann zeta function. Let A be the Glaisher-Kinkelin constant. Let finally $r_{2}(n)$ be the number of decomposition of the integer $n$ into the sum of the squares of two integers, and $\beta(n)=\sum_{n \geq 0} \frac{(-1)^{n}}{(2 n+1)^{s}}$ be the beta-Catalan (or betaDirichlet) function. Then

$$
\begin{aligned}
& \prod_{n \geq 1} n^{d(n)}=(2 \pi)^{-1 / 2} \\
& \prod_{n \geq 1} n^{\sigma(n)}=e^{1 / 24}(2 \pi)^{-1 / 24} A^{-1 / 2} \\
& \prod_{n \geq 1} n^{\varphi(n)}=(2 \pi e)^{1 / 6} A^{-2} \\
& \prod_{n \geq 1}^{d^{d^{2}(n)}}=(2 \pi)^{1 / 4} \\
& \prod_{n \geq 1}^{d\left(n^{2}\right)}=(2 \pi)^{-1 / 4} \\
& \prod_{n \geq 1} n^{\theta(n)}=1 \\
& \prod_{n \geq 1} n^{r_{2}(n)}=\Gamma(1 / 4) /\left(\pi^{1 / 4} \sqrt{2}\right) .
\end{aligned}
$$

Proof. Using, e.g., [9], we have:

$$
\begin{aligned}
& \sum_{n \geq 1} \frac{d(n)}{n^{s}}=\zeta^{2}(s), \text { for } \mathfrak{R} s>1 \\
& \sum_{n \geq 1} \frac{\sigma(n)}{n^{s}}=\zeta(s) \zeta(s-1), \text { for } \mathfrak{R} s>2 \\
& \sum_{n \geq 1} \frac{\varphi(n)}{n^{s}}=\frac{\zeta(s-1)}{\zeta(s)}, \text { for } \mathfrak{R} s>2 \\
& \sum_{n \geq 1} \frac{d^{2}(n)}{n^{s}}=\frac{\zeta^{4}(s)}{\zeta(2 s)}, \text { for } \mathfrak{R} s>1
\end{aligned}
$$

$$
\begin{aligned}
& \sum_{n \geq 1} \frac{d\left(n^{2}\right)}{n^{s}}=\frac{\zeta^{3}(s)}{\zeta(2 s)}, \text { for } \mathfrak{R} s>1 \\
& \sum_{n \geq 1} \frac{\theta(n)}{n^{s}}=\frac{\zeta^{2}(s)}{\zeta(2 s)}, \text { for } \mathfrak{R} s>1 \\
& \sum_{n \geq 1} \frac{r_{2}(n)}{n^{s}}=\zeta(s) \beta(s), \text { for } \mathfrak{R}(s)>1 .
\end{aligned}
$$

It is then easy to finish the proofs using the well-known values $\zeta(0)=-1 / 2, \zeta^{\prime}(0)=-\log (2 \pi) / 2, \zeta(-1)=-1 / 12$, $\zeta^{\prime}(-1)=-\log A+1 / 12$, as well as the possibly less-known values $\beta(0)=1 / 2$ and $\beta^{\prime}(0)=\log \left(\Gamma^{2}(1 / 4) / 2 \pi \sqrt{2}\right)$ which can be obtained from values of the Hurwitz zeta function $\zeta(s, u)=\sum_{n \geq 0} 1 /(n+u)^{s}$, namely $\zeta(0, u)=-u+1 / 2$, $\zeta^{\prime}(0, u)=\log (\Gamma(u) / \sqrt{2 \pi})$, since $\beta(s)=(\zeta(s, 1 / 4)-$ $\zeta(s, 3 / 4)) / 4^{s}$.

\section{Towards a notion of complexity of sequences of integers?}

It is tempting to try to use the zeta-regularized product of a non-decreasing sequence of integers $\lambda_{A}, \lambda_{2}, \ldots$ to "measure how complex" this sequence is. Does this product detect whether these numbers are "dense" or whether they tend quickly to infinity? One possible difficulty is that, in a usual (finite) product, there might be some "compensation": the product of "dense" numbers could be larger than the product of "scarce" numbers, but on the other hand scarce integers could grow extremely quickly making the product possibly huge. What happens with a zetaregularized product? An example given above is deceiving: the zeta-regularized product of the squarefree integers is equal to the zeta-regularized product of the squares.

Another approach could be to try to evaluate the tail of the zeta-regularized product: let $C_{k}(\Lambda)=\prod_{i=k+1}^{\infty} \lambda_{i}$. One has $C_{k}(\Lambda)=\left(\prod_{i=1}^{\infty} \lambda_{i}\right)\left(\prod_{j=1}^{k} \lambda_{j}\right)^{-1}$. This means that the zetaregularized product could discriminate between two sequences whose first $k$ terms give products with the same growth. But we have not found interesting examples yet.

On the other hand, let $\left(a_{n}\right)_{n}$ be the characteristic function of a sequence of integers $\Lambda$, and let us denote as previously $\zeta_{\Lambda}(s)=\sum_{n \in \Lambda} 1 / n^{s}=\sum_{n \geq 1} a_{n} / n^{s}$. The analytic density of $\Lambda$ is the limit (if it exists) of $\lim _{s \rightarrow 1}(s-1) \zeta_{\Lambda}(s)$ when $s$ tends to $1_{+}$. (Note that if $\zeta_{\Lambda}$ has a simple pole at $s=1$, then this limit is equal to $\operatorname{Res}_{s=1} \zeta_{\lambda}(s)$.) The logarithmic density of $\Lambda$ is the limit (if it exists) of $\left(\sum_{n \in \Lambda, n \leq x} 1 / n\right) / \log n$ when $n$ goes to infinity: a nice result is that one of these two densities exists for a sequence $\Lambda$ if and only if the other one exists, and they must be equal (see, e.g., [21, p. 96]). Is it reasonable to think that there might exist a sort of density associated with this residue (compare with Definition 2, where the quantity $\operatorname{Res}_{s=0} \frac{\zeta \Lambda(s)}{s^{2}}$ occurs), or is it totally hopeless to evaluate the scarcity/density of a sequence of integers from its zeta-regularized product? 


\section{References}

[1] J.-P. Allouche, The zeta-regularized product of odious numbers, Adv. in Appl. Math. (2019) to appear. https://doi.org/10.1016/j.aam.2019.101944.

[2] J.-P. Allouche, H. Cohen, Dirichlet series and curious infinite products, Bull. London Math. Soc. 17, 531538 (1985).

[3] J.-P. Allouche, M. Mendès France, J. Peyrière, Automatic Dirichlet series, J. Number Theory 81, 359373 (2000).

[4] J. Borwein, D. Bailey, R. Girgensohn, Experimentation in Mathematics: Computational Paths to Discovery (Taylor \& Francis, 2004).

[5] I. V. Blagouchine, Three notes on Ser's and Hasse's representations for the zeta-function, Integers $\mathbf{1 8 A}$, Paper $\sharp A 3$ (2018).

[6] C. Deninger, On the $\Gamma$-factors attached to motives, Invent. Math. 104, 245-261 (1991).

[7] U. K. Dutta, S. Sangeeta Pradhan, P. K. Ray, Regularized products over balancing and Lucas-balancing numbers, Indian J. Math. 60, 171-179 (2018).

[8] S. Egami, Some curious Dirichlet series, in Analytic number theory and its interactions with other parts of number theory (Kyoto, 1998), Sūrikaisekikenkyūsho Kōkyūroku 1091 (1999), 172-174.

[9] H. W. Gould, Temba Shonhiwa, A catalog of interesting Dirichlet series, Missouri J. Math. Sci. 20, 218 (2008).

[10] S. W. Hawking, Zeta function regularization of path integrals in curved spacetime, Comm. Math. Phys. 55, 133-148 (1977).

[11] K. Kimoto, M. Wakayama, Remarks on zeta regularized products, Int. Math. Res. Not. 2004, 855-875 (2004).

[12] K. Kimoto, N. Kurokawa, C. Sonoki, M. Wakayama, Some examples of generalized zeta regularized products, Kodai Math. J. 27, 321-335 (2004).

[13] A. R. Kitson, The regularized product of the Fibonacci numbers, Preprint (2006), electronically available at https://arxiv.org/abs/math/0608187.
[14] N. Kurokawa, M. Wakayama, A generalization of Lerch's formula, Czechoslovak Math. J. 54, 941-947 (2004).

[15] N. Kurokawa, M. Wakayama, Generalized zeta regularizations, quantum class number formulas, and Appell's $O$-functions, Ramanujan J. 10, 291-303 (2005).

[16] E. Landau, A. Walfisz, Über die Nichtfortsetzbarkeit einiger durch Dirichletsche Reihen definierter Funktionen, Rend. Circ. Mat. Palermo 44, 82-86 (1920).

[17] Y. Mizuno, Generalized Lerch formulas: examples of zeta-regularized products, J. Number Theory $\mathbf{1 1 8}$, 155-171 (2006).

[18] E. Muñoz García, R. Pérez Marco, The product over all primes is $4 \pi^{2}$, Comm. Math. Phys. 277, 69-81 (2008).

[19] L. Navas, Analytic continuation of the Fibonacci Dirichlet series, Fibonacci Quart. 39, 09-418 (2001).

[20] On-Line Encyclopedia of Integer Sequences, founded by N. J. A. Sloane, electronically available at http://oeis.org.

[21] H. Ostmann, Additive Zahlentheorie I, Ergebnisse der Math. u. ihrer Grenzgebiete, Springer, 1956.

[22] J. R. Quine, S. H. Heydari, R. Y. Song, Zeta regularized products, Trans. Amer. Math. Soc. 338, 213 231 (1993).

[23] S. S. Rout, G. K. Panda, Balancing Dirichlet series and related $L$-functions. Indian J. Pure Appl. Math. 45, (2014), 943-952 (2014).

[24] V. V. Smirnov, The $\zeta$-regularized product over all primes, Preprint (2015), electronically available at https://arxiv.org/abs/1503.06954.

[25] C. Soulé, D. Abramovich, J.-F. Burnol, J. Kramer, The determinant of Laplace operators, in Lectures on Arakelov Geometry, Cambridge Studies in Advanced Mathematics 33, Cambridge University Press, Cambridge, 1992, pp. 93-114.

[26] A. Sourmelidis, On the meromorphic continuation of Beatty Zeta-functions and Sturmian Dirichlet series, J. Number Theory 194, 303-318 (2019).

[27] G. N. Watson, Theorems stated by Ramanujan (VIII): Theorems on divergent series, J. London Math. Soc. 4, 82-86 (1929). 